\title{
Local Atomic Structural Mapping by EELS Spectral Imaging and EXELFS Analysis
}

Ping Lu and Paul Kotula,

Sandia National Laboratories, PO Box 5800, Albuquerque, NM 87185

Spectral imaging (SI) techniques that collect point-spectra at a two-dimensional array of points have been used widely to collect a full spectral datasets for comprehensive chemical microanalysis $[1,2]$. The common spectra used in scanning transmission electron microscopy (STEM) are energydispersive X-ray spectra (EDS) although use of the electron energy loss spectra (EELS) has also been demonstrated [3]. The SI dataset can be analyzed by multivariate statistical analysis (MSA) techniques beyond principal components analysis (PCA), providing us with statistically-derived compositional phase maps in complex multi-phase materials [2]. Extended electron energy loss fine structure (EXELFS) in EELS has been developed to determine the local atomic bonding structures of materials [4]. The technique, however, has not been widely utilized due to a low signal-to-noise $(\mathrm{S} / \mathrm{N})$ in extended energy loss range. The electron beam radiation damage to the sample is a serious issue due to a large electron dose required to improve the $\mathrm{S} / \mathrm{N}$ in the spectra.

In this work, we describe a technique for obtaining local atomic structural map of a composite based on: (1) collection of EELS SI dataset from a composite area; (2) MSA of EELS SI dataset to obtain maps of the EELS components and the pure components present within the composite area and (3) EXELFS analysis of the pure EELS components. The EELS-SI data collection consists of scanning a nano-electron probe over the specimen and recording EELS spectrum from each specimen position. In the MSA approach, the spatially-resolved EELS data matrix D is factored into two contributions, one containing the spectral characteristics (S) of the species and one contains their respective spatial abundances or scoring values $(\mathrm{C})$, which can be represented as the matrix product $\mathrm{D}=\mathrm{CS}^{\mathrm{T}}$, where the superscript $\mathrm{T}$ denotes a matrix transpose. This factorization can be performed by PCA, with use of the rotation by the VARIMAX procedure to maximize the spatial simplicity of $\mathrm{C}$ [5]. The MSA allows for determination of the principal components and 2-D scoring maps that describe the distribution of the components. In our method, the pure EELS components representative of the composites are obtained by averaging the spectra from the specimen locations (pixels) that show a similar scoring contribution in the 2-D scoring maps. This method allows us to obtain the high S/N EELS spectra which can be analyzed directly by EXELFS to obtain the local atomic structure [4]. Since the time of electron beam exposure at each specimen pixel in SI data collection is limited to the dwell time at each pixel, the radiation damage to the specimen can be largely avoided.

As an example of this approach, we examined a sample with mixture of BN particles and amorphous carbon film. The sample was made by grinding a BN bulk sample in methanol and collecting the BN particles on a holy-carbon TEM support film. The EELS-SI data were acquired by a FEI Tecnai F30STEM, operated at $300 \mathrm{kV}$ under the following conditions: an electron probe of about $10 \mathrm{~nm}$ diameter, diffraction camera length of $100 \mathrm{~mm}$, and GIF entrance aperture of $3 \mathrm{~mm}$, and dwell time of $400 \mathrm{~ms}$ at each pixel. Fig.la is an annular dark field STEM image of the sample, showing a square region of dimensions $200 \mathrm{~nm} \times 200 \mathrm{~nm}$ with sampling pixels of 20x20 used for EELS SI collection. The SI dataset consisted of 400 EELS spectra. Each EELS spectrum in SI dataset was filtered to remove abnormal intensity due to x-ray striking the CCD. The PCA eigenanalysis of EELS-SI dataset indicated there were 9 non-noise factors. The four of PCA components were apparently due to a systematical energy shift (zero-loss peak) as the electron beam was scanned across the region, and 
two were related to variation of specimen thickness. Fig.1b shows three most significant PCA components, along with respective scoring maps of the components (Figs.1c-1d). It is clear that the components 1, 2, and 3 correspond to EELS spectrum from the BN, BN/C overlapped area, and carbon area respectively. From the scoring maps, the EELS spectra from pixels with similar scoring values could be isolated. Figs.2a and $2 \mathrm{c}$ show the pure EELS spectra of the BN and amorphous carbon, obtained by averaging the EELS spectra from the pixels with scoring values of more than 0.9 in the maps of component 1 (Fig.1c) and 3 (Fig.1e), respectively. These two EELS spectra (Figs.2a and 2c) were used in our EXELFS analysis to obtain radial distribution function (RDF) of the BN and amorphous carbon, respectively [4]. The results, shown in Figs.2b and $2 \mathrm{~d}$ for BN and amorphous carbon, respectively, are consistent with those from previous works [6,7]. In conclusion, our technique provides the capability of 2-D structural mapping and avoids the radiation damage to the samples often associated with the EXELFS. The details of our method and its application to structural mapping of the composites will be reported in our future publication [8].

[1] G.J.F. Legge and I. Hammond, J. Microsc. 117, (1979) 201.

[2] P. Kotula et al., Microsc. Microanal. 9, (2003) 1.

[3] P. Kotula et al., Microsc. Microanal. 10, (2004) 874.

[4] R. F. Egerton, Electron Energy-Loss Spectroscopy in the Electron Microscope, $2^{\text {nd }}$ Ed. (1996) Plenum Press, New York

[5] M. Keenan, Surf. Interface Anal. 41, (2009) 79.

[6] Serin et al., Microsc. Micrianal. Microstruct. 3, (1992) 201.

[7] Moller et al., Appl. Surf. Sci.108, (1997) 59.

[8] Sandia is a multiprogram laboratory operated by Sandia Corporation, a Lockheed Martin Company, for the United Stated Department of Energy under contract DE-AC04 94AL 85000.
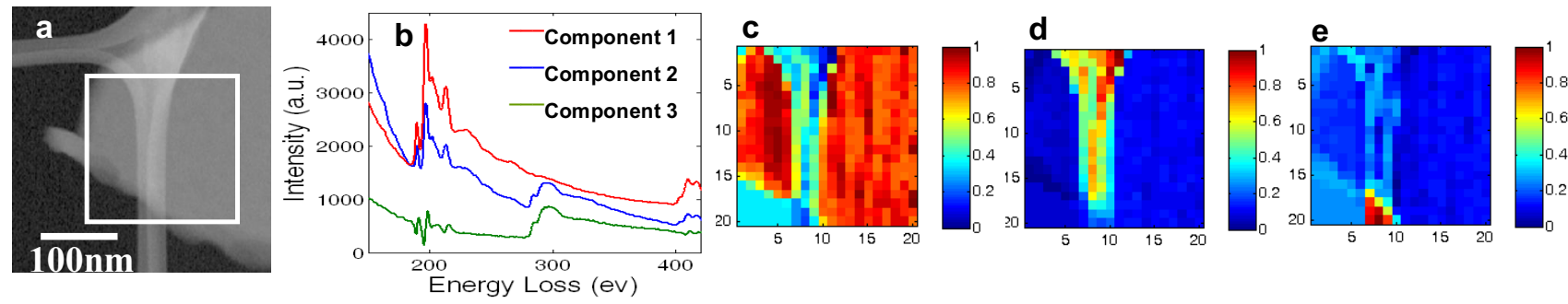

Fig.1. (a) A annular dark-field STEM image showing the area used for the analysis; (b) three components obtained from the PCA analysis; and scoring maps (c, d, and e) for each component.
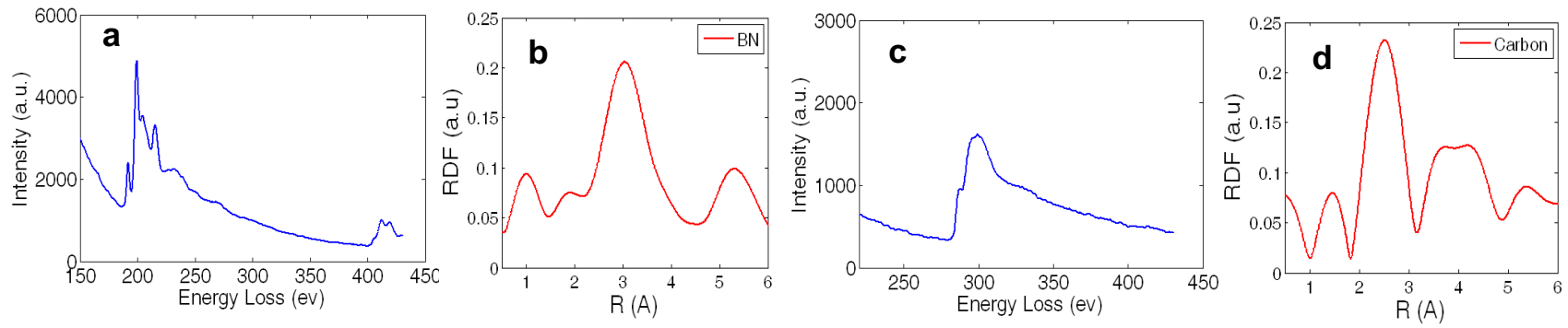

Fig.2. Pure EELS spectra for BN (a) and amorphous carbon (c), obtained by averaging EELS spectra from the locations with similar scoring values $(>0.9)$ in Fig.1c and Fig. 1e, respectively. RDFs for the BN (b) and amorphous carbon (d) were obtained by analysis of EELS spectra by EXELFS following the approach in Reference 4. 